\title{
¿Existe en América del Sur una brecha de consenso sobre el cambio climático? Evidencia a partir del análisis de percepción en redes sociales
}

\author{
FERNANDO A. I. GONZÁLEZ \\ Instituto de Investigaciones Económicas y Sociales del Sur.
}

\begin{abstract}
Resumen. Este trabajo indaga acerca de la posible existencia de una brecha de consenso entre la evidencia científica y la percepción pública mayoritaria, en relación con el cambio climático en América del Sur. Se utilizaron técnicas de minería de texto para extraer datos de la red social Twitter, georreferenciados en América del Sur, durante septiembre y octubre de 2019. El texto seleccionado fue clasificado a partir de dos clasificadores: el clasificador bayesiano ingenuo y el de máquinas de soporte vectorial. Ambos algoritmos presentaron tasas de precisión elevadas $(>80 \%)$. Los resultados sugieren que en la actualidad no existe brecha de consenso para el caso de América del Sur. Esta brecha parece estar restringida a países como Estados Unidos. En América del Sur, entre el $86 \%$ y $95 \%$ de todos los tweets se clasificó como positivo, es decir, que cree que el cambio climático es real.
\end{abstract}

[Palabras clave: opinión pública, ambiente, algoritmos de clasificación, Twitter]

\begin{abstract}
Is there a consensus gap on climate change in South America? Evidence from sentiment analysis in social networks. This work investigates the possible existence of a consensus gap between scientific evidence and majority public perception, related to climate change in South America. Text mining techniques were used to extract georeferenced data from the social network Twitter during September and October of 2019. The selected text is classified from two classifiers such as the naive Bayesian classifier and the vector support machines classifier. Both classification algorithms have high accuracy rates $(>80 \%)$. The results suggest the absence of a consensus gap in the case of South America, at present. This gap seems to be restricted to the case of countries like the United States. In South America, $86 \%$ to $95 \%$ of all tweets was classified as positive (believes that climate change is real).
\end{abstract}

[Keywords: public opinion, environment, classification algorithms, Twitter]

\section{INTRODUCCIÓN}

En años recientes, la comunidad científica ha manifestado un creciente consenso acerca de la existencia, causas y potenciales efectos negativos derivados del cambio climático (Oreskes 2004; Doran and Kendall Zimmerman 2009; Carlton et al. 2015) (Tabla 1). En este sentido, a partir de una revisión de literatura (Cook et al. 2013) surge que el 97\% de los científicos que estudiaron al cambio climático, y en particular al calentamiento global, coinciden en que este fenómeno existe y es causado por la actividad humana. Sin embargo, una cantidad significativa de personas manifiesta su escepticismoen relación con el cambio climático y sus consecuencias, o tiende a subestimar su importancia (Dunlap and Saad 2001; Leiserowitz 2005; Johnson and Levin 2009; Pew 2013). En efecto, a partir de una encuesta sobre cambio climático realizada en los Estados Unidos en el año 2013 se observó que apenas $49 \%$ de los encuestados creía que el

Editora asociada: Ana Cingolani cambio climático es causado por la actividad humana y sólo $38 \%$ sostuvo que el cambio climático afecta a las personas (Leiserowitz et al. 2013). A esto se lo denominó "brecha de consenso" entre la evidencia científica y la percepción pública (Reusswig 2013; Cook 2019).

El análisis de la brecha de consenso es relevante porque la percepción pública afecta de forma directa el proceso de formulación de políticas ambientales y porque la falta de apoyo de la ciudadanía puede retrasar o impedir que se implementen políticas de mitigación (Cook et al. 2013). El origen de esta brecha responde a múltiples causas: preferencias políticas —adeptos a partidos más conservadores tienden a rechazar medidas de regulación sobre la industria y descreen del cambio climático- (Campbell and Kay 2014), campañas negacionistas por parte de think tanks conservadores (Boussalis and Coan 2015), desconocimiento de la evidencia 
científica o uso de información errónea (Cook et al. 2013). En este sentido, y en el contexto de Sudamérica, la potencial existencia de una brecha de consenso es el interrogante principal que intenta responder este trabajo.

Las redes sociales se suelen utilizar para conocer la percepción de las personas sobre diversos temas, y a bajo costo. En particular, desde su lanzamiento en 2006, Twitter se convirtió en una red social que permite recolectar la percepción de las personas a escala global. Cuenta con más de 330 millones de usuarios activos cada mes, que diariamente generan 500 millones de tweets (mensajes) (Omnicore 2019). Las ciencias sociales aprovecharon la existencia de esta fuente de información. Numerosos trabajos avanzan en el análisis de percepción (del inglés, sentiment) empleando técnicas de minería de texto como búsquedas por palabras clave o hashtags en tweets. Algunos de las temáticas analizadas incluyen a la percepción sobre marcas comerciales (Jansen et al. 2009), reseñas de productos (Zagibalov and Carroll 2008), predicción de ventas (Rui et al. 2013) o de resultados electorales (Bermingham and Smealton 2010) o estimación de felicidad (Allisio et al. 2013). Otras plataformas empleadas en el análisis de percepción incluyen a Facebook (Rahman 2012; Ortigosa et al. 2014), foros online (Abbasi et al. 2008) o artículos periodísticos (Taj et al. 2019). Como es lógico, las opiniones vertidas en estas redes no necesariamente representan las de la población general. En promedio, los usuarios de redes sociales son más jóvenes y tienen mayor educación e ingresos que los no-usuarios (Pew 2019), aunque al controlar por edad, género y educación no se observan diferencias significativas con los no-usuarios en cuanto a creencias y preferencias políticas (Mellon and Prosser 2017).

Recientemente, Twitter también se ha usado para examinar la percepción sobre el cambio climático (An et al. 2014; Cody et al. 2015; Jang and Hart 2015). Cody et al. (2015) realizaron un análisis de percepción a partir de palabras clave de tweets en inglés y hallaron quealgunos hechos, como desastres naturales, tienden a incrementar la negatividad percibida sobre el cambio climático. An et al. (2014) sostuvieron que los desastres naturales reducen el escepticismo sobre el cambio climático y que, la información de Twitter es una herramienta apropiada para reflejar la percepción pública y sus cambios en el tiempo. Jang y Hart (2015) exploraron las amplias diferencias en cómo perciben el cambio climático distintos países de habla inglesa; lo hicieron a partir de buscar por palabras clave en Twitter, y concluyeron que algunos estados de EE.UU. presentan el mayor grado de escepticismo - en particular, aquellos con mayor proporción de votantes del partido republicano-, mientras que un menor nivel de escepticismo se observa en Canadá, Australia y el Reino Unido.

A su vez, Mucha (2017) evaluó la percepción de las personas (positiva [cree que el cambio climático es verdad], negativa [cree que es falso] y neutra [ninguna de las anteriores]) usando técnicas como el clasificador bayesiano ingenuo; este autor halló que hacia 2014, la cantidad de personas con una percepción positiva sobre el cambio climático superaba a las de percepción negativa, aunque las primeras mostraban una tendencia decreciente. Dahal et al. (2019) exploraron diferencias temporales y geográficas en las percepciones sobre cambio climático y encontraron que una mayor frecuencia de días cálidos en otoño o invierno tiende a reducir el escepticismo. En otros trabajos sobre el tema se analizó el efecto del clima sobre los estados de ánimo reflejados en tweets (Yang et al. 2015), el impacto de una celebridad hablando sobre el clima (Leas et al. 2016), las diferencias de género en la percepción (Holmberg and Hellsten 2015) o los patrones diarios y semanales de tweets sobre cambio climático (Kirilenko and Stepchenkova 2014). En particular, Leas et al. (2016) demuestran que luego de un célebre discurso de Leonardo Di Caprio referido al cambio climático, la cantidad de tweets relacionados aumentó 636\% respecto de los días previos, aunque no se exploró la percepción que contenían esos tweets.

En casi todos los casos analizados (excepto Kirilenko and Stepchenkova 2014), la recolección de tweets se realizó considerando sólo textos en idioma inglés. Sin embargo, esto podría no representar la percepción acerca del cambio climático en algunas regiones (Dahal et al. 2019) como, por ejemplo, América del Sur, donde el portugués y el español son los idiomas preponderantes.

Este trabajo busca analizar la existencia de una brecha de consenso en América del Sur en relación con el cambio climático, a través del examen de la percepción de las personas. Para ello, se hace uso de técnicas de minería de texto con datos de la red social Twitter y a partir de la búsqueda por palabras claves. Para ello, se consideran tweets georreferenciados a 
la región y originados en español o portugués. El período bajo estudio comprende los meses de septiembre y octubre de 2019. Este trabajo constituye un antecedente único en analizar un gran volumen de datos de redes sociales para evaluar la percepción acerca del cambio climático en América del Sur.

\section{Materiales y MéTOdos}

La selección de tweets relevantes se realizó empleando la interfaz de programación de aplicaciones (API, por sus siglas en inglés) de Twitter. Mediante palabras claves, se identificó a aquellos que contenían las expresiones "cambio climático" o "calentamiento global" (o sus equivalentes en portugués, mudança climática o aquecimento global). Además, para ser seleccionado, un tweet debía contener coordenadas geográficas que lo localizaran en América del Sur, o bien la localización del usuario que generó el tweet, según su perfil público, debía situarse en la región. El período de análisis abarcó desde el 14 de septiembre de 2019 hasta el 14 de octubre de 2019 y comprendió un total 43138 tweets relevantes.

Debe reconocerse que una amplia cantidad de tweets no posee coordenadas geográficas ni los perfiles de los usuarios información sobre su localización. Esto limita la cantidad de tweets que son seleccionados. Al respecto, no existe evidencia previa respecto a diferencias con tweets sin geolocalización. Esto significa que la elección de geolocalizar tweets no correlaciona con la percepción expresada en éstos. Además, los tweets geolocalizados constituyen una muestra razonablemente grande en este trabajo.

Los tweets recolectados se deben filtrar y homogeneizar antes de ser clasificados. Al respecto, se eliminaron los retweets para evitar duplicaciones de información. También se eliminaron del texto de cada tweet direcciones URL, nombres de usuario, signos de puntuación y palabras vacías (del inglés, stop words). Lógicamente, también se desecharon las palabras claves con las que se recolectaron los tweets. Por último, todo el texto se transformó a letras minúsculas para facilitar la comparación entre palabras.

Cada tweet seleccionado fue clasificado en positivo (la persona percibe al cambio climático como verdadero), negativo (la persona percibe al cambio climático como falso) o neutral (ninguno de los anteriores). Así, la muestra de tweets permite concluir acerca de la percepción regional sobre el cambio climático y la posible brecha de consenso respecto a la evidencia científica. La clasificación de tweets se realizó empleando dos algoritmos de aprendizaje de máquinas muy utilizados en la minería de textos. Por una parte, se recurrió al clasificador bayesiano ingenuo, basado en el teorema de Bayes (Bayes 1763) y el supuesto de independencia de características relevantes, que se desempeña de forma adecuada en la clasificación de grandes volúmenes de textos (An et al. 2014; Mucha 2017). Por otra parte, se empleó el clasificador de máquinas de soporte vectorial (del inglés, support vector machines) (Boser et al. 1992), que separa las categorías de interés al encontrar el hiperplano que maximiza la distancia entre ellas. Se empleó una especificación de kernel lineal. Para ambos algoritmos, el procedimiento de clasificación se aplicó de la misma forma, según se detalla a continuación.

A los efectos de la clasificación se seleccionaron aleatoriamente 10000 tweets de la muestra y se procedió a clasificarlos en positivos, negativos o neutros. Estos tweets constituyeron las muestras de entrenamiento (con las cuales los clasificadores "aprenden" a clasificar) y de prueba (con la cual se evalúa la precisión de los clasificadores). La clasificación -de la submuestra de 10000 tweets- se realizó en función de palabras clave en el texto de cada tweet, siguiendo la propuesta de Jang y Hart (2015). Estos autores proponen agrupar tweets de acuerdo con la presencia de palabras claves en su contenido. De esta forma, si el texto de un tweet contenía palabras como "real", "verdadero" o "hecho", al tweet se lo clasificaba como positivo, mientras que la aparición de palabras como "falso", "farsa" o "mentira" implicaba que al tweet se lo clasificaba como negativo. Lógicamente, este criterio está sujeto a imprecisiones (i.e., un tweet del tipo "el cambio climático no es real" es clasificado como positivo cuando no lo es), aunque demostró generar clasificaciones con una elevada precisión al analizar grandes volúmenes de datos (Jang and Hart 2015). Los tweets no clasificados según el criterio previo (14\% de la submuestra) fueron clasificados de forma manual al no contener ninguna de las palabras clave consideradas. El $81 \%$ —nuevamente, de la submuestra de 10000 tweets - resultó clasificado como positivo, el $11 \%$ como neutro y el $8 \%$ restante como negativo.

De la anterior sub-muestra de 10000 tweets, $70 \%$ fue seleccionado como muestra 
de entrenamiento y $30 \%$ como muestra de prueba. Al entrenar al clasificador bayesiano ingenuo con la muestra de entrenamiento y luego utilizarlo para predecir la clasificación de cada tweet en la muestra de prueba se obtuvo una clasificación correcta (tasa de precisión) del 79.6\% de los casos. Al emplear el algoritmo de clasificación de máquinas de soporte vectorial la tasa de precisión ascendió al $88.16 \%$.

Los tweets restantes (33138) son clasificados por ambos algoritmos de clasificación (bayesiano y SVM) luego de su etapa de entrenamiento y prueba. Estos clasificadores constituyen, esencialmente, métodos de aprendizaje de máquina (del inglés, machine learning) en los que los algoritmos aprenden a desarrollar una tarea (etapa de entrenamiento). Sus resultados son evaluados mediante la tasa de precisión (etapa de prueba). Finalmente, los algoritmos entrenados predicen la clasificación de las observaciones. Si todos los tweets estuvieran previamente clasificados, usar estos clasificadores carecería de razón. Además, la clasificación —según se detalló en los párrafos precedentes - requiere la intervención humana (clasificación manual), aunque se busca reducirla al mínimo porque es una fuente potencial de sesgo y porque demanda mucho tiempo.

Además de usar algoritmos de clasificación, el análisis de percepción se complementó con el análisis de palabras y pares más frecuentes (bigramas). Esto permite conocer cuáles fueron las palabras más mencionadas en los tweets seleccionados y, por ende, delimitar con qué fenómenos se asocia frecuentemente al cambio climático. El procesamiento y análisis de datos se realizó en Python 3.7. Se recurrió especialmente a librerías como tweepy, pandas, numpy, nltk (siglas para Natural Language Processing Tool Kit) y sklearn.

\section{Resultados}

Las palabras más frecuentes en tweets pertenecientes a la muestra de análisis se presentan en la Figura 1. Esto permite conocer, como una primera aproximación, con qué fenómenos se asocia la percepción del cambio climático en la región.

Las palabras más utilizadas reflejan una tendencia a percibir el cambio climático como un fenómeno de escala global (palabras "planeta", "mundo", "terra"), que debe ser atendido ("frenar", "mitigar", "detener", "agora") y que puede generar consecuencias negativas ("matar", "estragos") (Figura 1). También es posible examinar la frecuencia de la ocurrencia simultánea de las palabras contenidas en tweets (bigramas). La Tabla 1 presenta los resultados tabulados para las combinaciones de palabras más comunes, en español y portugués.

De la Tabla 2 se desprenden ciertos puntos en común en la región: palabras como "matarmatarnos", "planeta-mundo" y "mitigardetener" aparecen entre los pares más frecuentes. En idioma español, una mayor cantidad detweets hacen uso dela combinación
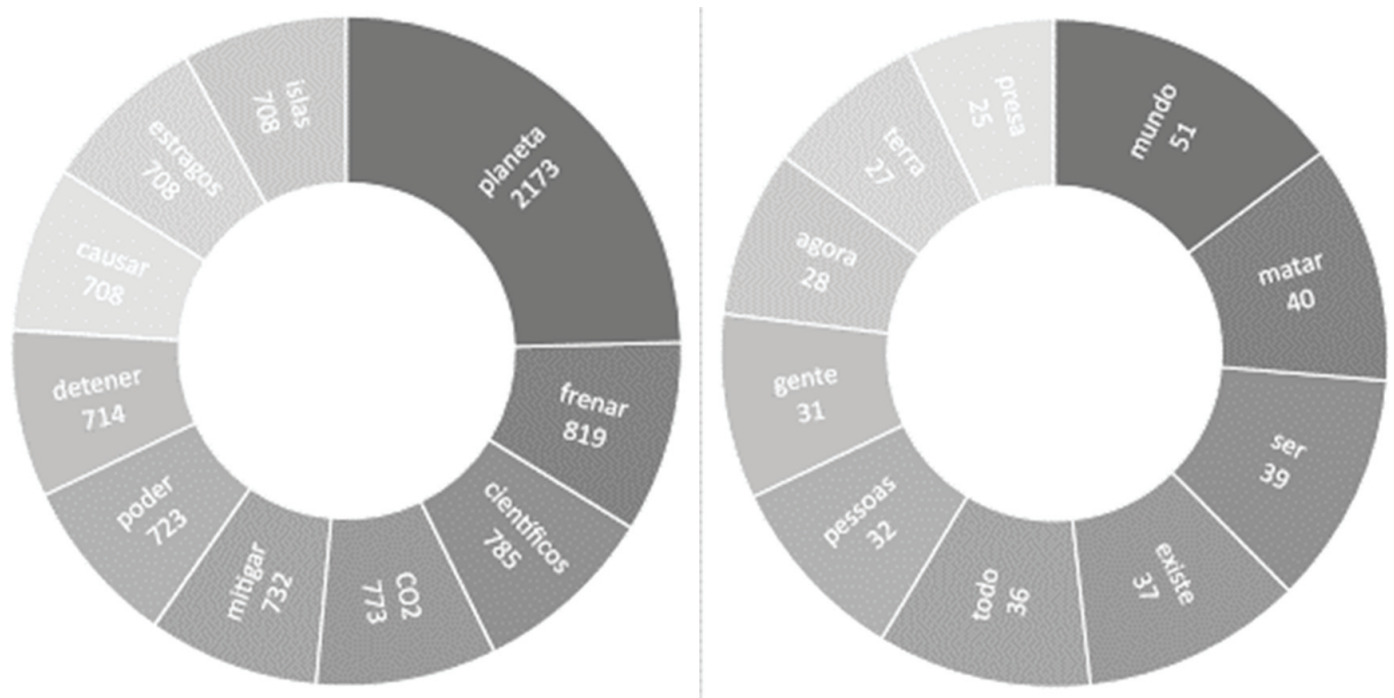

Figura 1. Diez palabras más frecuentes en tweets seleccionados, en español (izquierda) y portugués (derecha).

Figure 1. Ten most frequent words in selected tweets, in Spanish (left) and Portuguese (right). 
Tabla 1. Existencia, causas y potenciales consecuencias del cambio climático.

Table 1. Existence, causes and potential consequences of climate change.

\begin{tabular}{lll}
\hline & Causas & Consecuencias \\
\hline $\begin{array}{l}\text { Aumento de la temperatura global } \\
\text { promedio (frecuentemente comparada con } \\
\text { la temperatura en la etapa pre-industrial) }\end{array}$ & $\begin{array}{l}\text { Emisión de gases de efecto invernadero } \\
\text { dióxido de carbono o metano) }\end{array}$ & $\begin{array}{l}\text { Incremento en la frecuencia } \\
\text { naturales (especialmente } \\
\text { inundaciones, sequías, } \\
\text { incendios y olas de calor) }\end{array}$ \\
$\begin{array}{lll}\text { Aumento en la variabilidad de las } \\
\text { precipitaciones (en exceso y en defecto }\end{array}$ & Deforestación & $\begin{array}{l}\text { Aumento del nivel del mar } \\
\text { Incremento de enfermedades } \\
\text { infecciosas }\end{array}$ \\
& & Pérdida de biodiversidad \\
\hline
\end{tabular}

Fuente: IPCC (2018)

Tabla 2. Bigramas de palabras más frecuentes en tweets sobre cambio climático, por idioma.

Table 2. Bigrams of words in climate change tweets, by language.

\begin{tabular}{cll}
\hline Orden & Español & Portugués \\
\hline 1 & (remedio, enfermedad) & (jane, fonda) \\
2 & (falsos, mitos) & (vai, matar) \\
3 & (proponen, detener) & (todo, mundo) \\
4 & (poder, mitigar) & (roupas, passar) \\
5 & (matarnos, planeta) & (plásticos, poluição) \\
\hline
\end{tabular}

Tabla 3. Clasificación de tweets y percepción del cambio climático en América del Sur ( $n=33138$ tweets).

Table 3. Classification of tweets and perception of climate change in South America ( $n=33138$ tweets).

\begin{tabular}{lccc}
\hline $\begin{array}{l}\text { Algoritmo de } \\
\text { clasificación }\end{array}$ & Positivo & Negativo & Neutro \\
\hline $\begin{array}{l}\text { Clasificador } \\
\text { bayesiano } \\
\text { ingenuo }\end{array}$ & 95.39 & 4.50 & 0.11 \\
$\begin{array}{l}\text { Máquinas de } \\
\text { soporte vectorial }\end{array}$ & 86.98 & 5.27 & 7.75 \\
\hline
\end{tabular}

"falsos" y "mitos", mientras que en portugués cobra una mayor relevancia la combinación "jane" y "fonda", en alusión a la detención de la actriz estadounidense Jane Fonda en el marco de una protesta por el cambio climático. Por último, este ejercicio de combinación de palabras frecuentes puede ser reiterado para cualquier orden. Así, al considerar tríos de palabras surgen combinaciones frecuentes como "americalatina-capital-biodiversidad" o "biodiversidad-motor-desarrollo". Por su parte, al emplear ambos algoritmos para predecir la clasificación de la muestra completa de tweets, más del 86\% de éstos se clasifica como positivo, aunque con diferencias entre los clasificadores (Tabla 3).

Al usar los dos algoritmos de clasificación surge que es mayoría el grupo de tweets que reconoce al cambio climático como real o verdadero (Tabla 3). Este resultado confirma la inexistencia de una brecha de consenso entre lo sugerido por la evidencia científica y la percepción pública mayoritaria, para el caso de América del Sur. Sin embargo, se observan diferencias significativas entre ambos algoritmos de clasificación: el clasificador bayesiano ingenuo parece subestimar la proporción de tweets neutrales,

Tabla 4. Brecha de consenso en antecedentes relevados. TW: Twitter.

Table 4. Consensus gap in the literature. TW: Twitter.

$\begin{array}{lllclcc}\text { Autor } & \text { País/Región } & \text { Período } & \text { Fuente } & \text { Método } & \begin{array}{c}\text { Perc. } \\ \text { positiva }\end{array} & \begin{array}{c}\text { Perc. } \\ \text { negativa }\end{array} \\ \begin{array}{l}\text { An et al. } \\ (2014)\end{array} & \begin{array}{l}\text { Todos los de habla } \\ \text { inglesa }\end{array} & \begin{array}{l}\text { Octubre a } \\ \text { diciembre } 2013\end{array} & \text { TW } & \begin{array}{l}\text { Clasificador bayesiano y } \\ \text { de máquinas de soporte } \\ \text { vectorial }\end{array} & 85 \% & 15 \% \\ \begin{array}{l}\text { Mucha } \\ (2017)\end{array} & \begin{array}{l}\text { Estados Unidos, } \\ \text { Reino Unido, } \\ \text { Australia y } \\ \text { Canadá }\end{array} & 2017 & \text { TW } & \begin{array}{l}\text { Clasificador de } \\ \text { máquinas de soporte } \\ \text { vectorial }\end{array} & 53 \% & 40 \% \\ \begin{array}{l}\text { Presente } \\ \text { estudio }\end{array} & \begin{array}{l}\text { América del Sur } \\ \text { Septiembre a }\end{array} & \text { TW } & \begin{array}{l}\text { Clasificador bayesiano y } \\ \text { de máquinas de soporte } \\ \text { vectorial }\end{array} & 86-95 \% & 5-4 \% \\ \end{array}$


a la vez que sobreestima aquellos positivos. Los resultados del clasificador de máquinas de soporte vectorial, mostraron una mayor tasa de precisión en la etapa de prueba y por ende sus resultados son más confiables.

Los resultados anteriores se muestran más positivos que estimaciones previas en la temática (Tabla 4). Así, An et al. (2014) hallan que más del $80 \%$ de los tweets recolectados para una muestra de países de habla inglesa creen en el cambio climático. Mucha (2017) afirma, para el caso estadounidense, que el $53 \%$ de todos los tweets es clasificado como positivo en el año 2017.

\section{Discusión y CONCLUSIONES}

A lo largo de este trabajo se intentó responder al interrogante de la posible existencia de una brecha de consenso sobre el cambio climático en relación con la evidencia científica y la percepción pública mayoritaria, a partir del análisis de la red social Twitter. Los antecedentes examinados mostraron un uso casi exclusivo de tweets en idioma inglés al analizar la cuestión de la percepción del cambio climático. Lógicamente, los resultados de estos trabajos pueden no ser representativos en el contexto de América del Sur, donde los idiomas predominantes son el español y el portugués.

Al recolectar tweets georreferenciados de la región de interés y emplear dos algoritmos de clasificación muy difundidos en minería de textos, no se halló evidencia consistente con la idea de una brecha de consenso para América del Sur. Los anteriores resultados de clasificación son algo mayores a lo reportado en antecedentes en la temática. Las diferencias en las estimaciones pueden deberse al período analizado (2019 en este trabajo versus 2013 y 2017 en los dos antecedentes reportados en la Tabla 4), a la cobertura geográfica de los tweets seleccionados (países de América del Sur versus países de habla inglesa o un sub-grupo de éstos) o en las categorías contempladas (An et al. [2014] no consideran la categoría neutro). De todas formas, las estimaciones de este trabajo (86-95\% de tweets positivos) se muestran concordantes con las de An et al. (2014) (85\% de tweets positivos) y con la proporción de científicos que considera que el cambio climático existe y es resultado de la actividad humana (97\%) (Cook et al. 2013).

Considerando los antecedentes examinados y lo reportado en este trabajo, es razonable concluir que la existencia de una brecha de consenso en el ámbito del cambio climático parece restringirse al caso de algunos estados de Estados Unidos (An et al. 2014), y en particular a aquellos con mayoría de votantes del partido republicano (Jang and Hart 2015). Esta brecha de consenso para el caso estadounidense también se ve reflejada en la forma en que periodistas presentan hechos relacionados al cambio climático -más enfocados en controversias políticas respecto al potencial impacto-, pero en menor medida en países como Alemania o Canadá (Tschotschel et al. 2020).

Lo anterior, sin embargo, debe examinarse en el marco de las limitaciones existentes. En primer lugar, las estimaciones aquí reportadas se construyen sobre tweets geolocalizados; esto es, una muestra de todos los tweets de interés. En segundo lugar, la API de Twitter impone limitaciones estrictas en la recolección de tweets. En cada momento, apenas es posible extraer tweets geolocalizados de hasta 7 días hacia atrás en la versión gratuita (y de hasta 30 días en la versión paga). A su vez, el período analizado abarca la realización de la Cumbre de Acción Climática 2019 (23 de septiembre), en la cual alcanzó amplia difusión la figura de Greta Thunberg.

Lógicamente, las limitaciones mencionadas pueden generar sesgos en las estimaciones. El período examinado, y en particular la figura de Greta Thunberg, puede dar lugar a un significativo incremento en la cantidad de tweets referidos a la temática - en concordancia con lo reportado por Leas et al. (2016) - , aunque no existe evidencia respecto a diferencias entre la distribución de esta mayor cantidad de tweets y la distribución de tweets observada en una semana normal.

Resulta relevante robustecer este análisis en el futuro, ampliando la cobertura temporal de tweets e intentando identificar el posible efecto que, sobre la percepción del cambio climático, poseen ciertos eventos como desastres naturales, declaraciones de personas influyentes y nuevos descubrimientos científicos. También es pertinente explotar las diferencias idiomáticas (portugués versus español) en la percepción del cambio climático. Finalmente, se concibe como útil la incorporación de otros criterios de clasificación de tweets (en las etapas de entrenamiento y prueba) — que no empleen simples palabras clave-, sino que consideren la semántica del texto a los efectos de reducir potenciales errores de clasificación. 


\section{REFERENCIAS}

Abbasi, A., H. Chen, and A. Salem. 2008. Sentiment Analysis in Multiple Languages: Feature Selection for Opinion Classification in Web Forums. ACM Trans. on Information Systems 26(3):1-34. https://doi.org/10.1145/ 1361684.1361685 https://doi.org/10.1145/1344411.1344413.

Allisio, L., V. Mussa, C. Bosco, V. Patti, and G. Ruffo. 2013. Felicittà: Visualizing and Estimating Happiness in Italian Cities from Geotagged Tweets. Working paper presented at 1st International Workshop on Emotion and Sentiment in Social and Expressive Media: Approaches and Perspectives from AI. URL: http://ceur-ws.org/Vol-1096/paper8.pdt

An, X., A. R. Ganguly, Y. Fang, S. B. Scyphers, A. M. Hunter, and J. G. Dy. 2014. Tracking climate change opinions from twitter data. Working paper presented at Workshop on Data Science for Social Good. URL: https://tinyurl.com/ y7umehu8.

Bayes, T. 1763. An essay towards solving a problem in the doctrine of chances. By the late Rev. Mr. Bayes, F. R. S. communicated by Mr. Price, in a letter to John Canton, A. Philosophical Transactions Royal Society 53. https:// doi.org/10.1098/rstl.1763.0053.

Bermingham, A., and A. Smeaton. 2010. Classifying Sentiment in Microblogs: Is Brevity an Advantage? Proc. of ACM CIKM Conf. Pp. 1833-1836. URL: https://core.ac.uk/download/pdf/147599841.pdf

Boser, B. E., I. M. Guyon, and V. N. Vapnik. 1992. A Training Algorithm for Optimal Margin Classifiers. Proceedings of the 5th Annual Workshop on Computational Learning Theory (COLT'92). Pp. 144-152. https://doi.org/10.1145/ 130385.130401.

Boussalis, C., and T. G. Coan. 2015. Text-mining the signals of climate change doubt. Global Environmental Change 36:89-100. https://doi.org/10.1016/j.gloenvcha.2015.12.001.

Campbell, T. H., and A. C. Kay. 2014. Solution aversion: on the relation between ideology and motivated disbelief. Journal of Personality and Social Psichology 107(5):809-824. https://doi.org/10.1037/a0037963.

Carlton, J. S., R. Perry-Hill, M. Huber, and L. S. Prokopy. 2015. The climate change consensus extends beyond climate scientists. Environmental Research Letters 10(9):1-12. https:/ / doi.org/10.1088/1748-9326/10/9/094025.

Cody, E. M., A. J. Reagan, L. Mitchell, P. S. Dodds, and C. M. Danforth. 2015. Climate change sentiment on twitter: An unsolicited public opinion poll. PloS one 10(8). https:/ / doi.org/10.1371/journal.pone.0136092.

Cook, J. 2019. Understanding and countering misinformation about climate change. Pp. 281-306 in I. Chiluwa and S. Samoilenko (eds.). Handbook of Research on Deception, Fake News, and Misinformation Online. IGI-Global: Hershey.

Cook, J., D. Nuccitelli, S. A. Green, M. Richardson, B. Winkler, R. Painting, R. Way, P. Jacobs, and A. Skuce. 2013. Quatifying the consensus on anthropogenic global warning in the scientific literature. Environmental Research Letters 8(2):1-8. https://doi.org/10.1088/1748-9326/8/2/024024.

Dahal, B., S. A. P. Kumar, and Z. Li. 2019. Topic modelling and sentiment analysis of global climate change tweets. Social Network Analysis and Mining 9(24):1-20. https:/ / doi.org/10.1007/s13278-019-0568-8.

Doran, P. T., and M. Kendall Zimmerman. 2009. Examining the scientific consensus on climate change. Eos, Transactions, American Geophysical Union 90(3). https://doi.org/10.1029/2009EO030002.

Dunlap, R. E., and L. Saad. 2001. Only one in four americans are anxious about the environment. Encuesta Gallup. URL: https://tinyurl.com/ydafmjuj.

Holmberg, K., and I. Hellsten. 2015. Gender differences in the climate change communication on Twitter. Internet Research 25(5):811-828. https:/ / doi.org/10.1108/IntR-07-2014-0179.

IPCC. 2014. Climate Change 2014: Impacts, Adaptation, and Vulnerability. Part A: Global and Sectoral Aspects. Contribution of Working Group II to the Fifth Assessment Report of the Intergovernmental Panel on Climate Change. URL: https://www.ipcc.ch/report/ar5/wg2/

IPCC. 2018. Impacts of $1.5^{\circ} \mathrm{C}$ Global Warming on Natural and Human Systems. In V. Masson-Delmotte, P. Zhai, H.-O. Pörtner, D. Roberts, J. Skea, et al. (eds.). Global Warming of $1.5^{\circ} \mathrm{C}$. URL: https://www.ipcc.ch/sr15/.

Jang, S. M., and P. S. Hart. 2015. Polarized frames on climate change and global warming across countries and states: Evidence from twitter big data. Global Environmental Change 32:11-17. https://doi.org/10.1016/ j.gloenvcha.2015.02.010.

Jansen, B. J., M. Zang, K. Sobel, and A. Chowdury. 2009. Twitter power: tweets as electronic word in mouth. Journal of the American Society for Information Science and Technology 60(11):2169-2188. https:/ /doi.org/10.1002/asi.21149.

Johnson, D., and S. Levin. 2009. The tragedy of cognition: psychological biases and environmental inaction. Currently Science 97(11):1593-1603.

Kirilenko, A., and S. Stepchenkova. 2014. Public microblogging on climate change: one year of Twitter worldwide. Global Environmental Change 26:171-182. https://doi.org/10.1016/j.gloenvcha.2014.02.008.

Leas, E. C., B. M. Althouse, M. Dredze, N. Obradovich, J. H. Fowler, S. M. Noar, J. Allem, and J. W. Ayers. 2016. Big Data Sensors of Organic Advocacy: The Case of Leonardo DiCaprio and Climate Change. PLoS ONE 11(8):1-9. https: //doi.org/10.1371/journal.pone.0159885.

Leiserowitz, A. A. 2005. American risk perceptions: is climate change dangerous? Risk Analysis 25(6):1433-1442. https: //doi.org/10.1111/j.1540-6261.2005.00690.x.

Leiserowitz, A. A., E. Maibach, C. Roser-Renouf, G. Feinberg, and P. Howe. 2013. Climate change in the American mind: Americans' global warming beliefs and attitudes in April, 2013. Yale University and George Mason University 
New Haven, CT: Yale Project on Climate Change Communication. https://doi.org/10.2139/ssrn.2298705.

Mellon, J., and C. Prosser. 2017. Twitter and Facebook are not representative of the general population: political attitudes and demographics of British social media users. Research and Politics, 4(3):1-9. https://doi.org/10.1177/ 2053168017720008

Mucha, N. 2017. Sentiment analysis of global warming using twitter data. Master thesis in Computer Science, North Dakota University. URL: https://tinyurl.com/yba8j79x.

Omnicore Agency. 2019. Twytter by the numbers 2018: stats, demographics and fun facts. URL: https:// www.omnicoreagency.com/twitter-statistics/

Oreskes, N. 2004. Beyond the ivory tower: The scientific consensus on climate change. Science 306:1686-1686. https: //doi.org/10.1126/science.1103618.

Ortigosa, A., J. M. Martin, and R. M. Carro. 2014. Sentiment Analysis in Facebook and its Application to ELearning. Computers in Human Behavior 31:527-541. https:/ / doi.org/10.1016/j.chb.2013.05.024.

Pew Research Center for the People and the Press. 2013. Public priorities: deficit rising, terrorism slipping. Informe URL: https:/ / tinyurl.com/y9qmdxce.

Pew Research Center for the People and the Press. 2019. Sizing up Twitter users. URL: https://tinyurl.com/ v3233b9.

Rahman, M. M. 2012. Mining social data to extract intellectual knowledge. arXiv preprint arXiv:1209.5345. https: / / doi.org/10.5815/ijisa.2012.10.02.

Reusswig, F. 2013. HIstory and future of the scientific consensus on anthropogenic global warning. Environmental Research Letters 8:1-4. https://doi.org/10.1088/1748-9326/8/3/031003.

Rui, H., Y. Liu, and A. Whinston. 2013. Whose and What Chatter Matters? The Effect of Tweets on Movie Sales. Decision Support Systems 55(4):863-870. https:/ / doi.org/10.1016/j.dss.2012.12.022.

Taj, S., A. Meghji, and B. B. Shaikh. 2019. Sentiment analysis of news articles: a lexicon-based approach. Working paper presented in $2^{\text {nd }}$ International Conference on Computing Mathematics and Engineering Technologies. https: //doi.org/10.1109/ICOMET.2019.8673428.

Tschotschel, R., A. Schuck, and A. Wonneberger. 2020. Patterns of controversy and consensus in German, Canadian and US online news on climate change. Global Environmental Change 60:1-12. https://doi.org/10.1016/ j.gloenvcha.2019.101957.

Yang, W., L. Mu, and Y. Shen. 2015. Effect of climate and seasonality on depressed mood among twitter users. Applied Geography 63:184-191. https://doi.org/10.1016/j.apgeog.2015.06.017.

Zagibalov, T., and J. Carroll. 2008. Unsupervised classification of sentiment and objectivity in Chinese text. Working paper presented at the Third International Joint Conference on Natural Language Processing: Volume-I. URL: https: L/www.aclweb.org/anthology/I08-1040/. 